\title{
NOVO MÉTODO “UPLOT-DAP” PARA REPRESENTAÇÃO GRÁFICA DE DISTRIBUIÇÕES DIAMÉTRICAS
}

\author{
Ulisses Silva da Cunha ${ }^{1}$ \\ Sebastião do Amaral Machado ${ }^{2}$ \\ Afonso Figueiredo Filho ${ }^{3}$ \\ Roberto Tuyoshi Hosokawa ${ }^{4}$
}

\begin{abstract}
RESUMO
Foi apresentado um novo método gráfico denominado de "UPLOT-DAP" cuja finalidade é auxiliar a preparação de dados para interpretação visual ou análises sobre o comportamento de variáveis por classes diamétricas. A pesquisa objetivou desenvolver uma solução gráfica para a determinação das classes diamétricas. Para desenvolver e testar o método foram utilizados 28320 DAP com casca de árvores procedentes de 15 blocos de 96ha da Floresta Nacional do Tapajós, Belterra, Pará. O "UPLOT-DAP" é bastante flexível e utiliza-se de técnicas de análise visual de dados como forma de interpretação da dispersão dos diâmetros. Os gráficos foram construídos considerando uma amplitude de classe diamétrica de $10 \mathrm{~cm}$ adotada como valor de referência. Foram escolhidos 2 blocos com amplitudes em DAP $(\mathrm{cm})$ representativas da população, bloco 18 [55,225] e bloco $25[55,195]$ para discutir os resultados. O principal papel do novo método é evitar a construção equivocada de perfis diamétricos inexistentes ou diferentes da realidade. A eficiência do "UPLOT-DAP" baseia-se na sua capacidade de controlar a variação dos dados de modo que as análises sejam mais consistentes com os fatores físicos e biológicos que caracterizam a dinâmica de florestas naturais. Através do uso de "UPLOT-DAP" foi possível também mostrar que a tentativa de se fixar uma amplitude constante ou com variação constante crescente para os dados, pode resultar em conclusões duvidosas sobre a estrutura diamétrica da floresta.

Palavras-Chave: Classe diamétrica, Método gráfico, Floresta tropical
\end{abstract}

\section{NEW METHOD “UPLOT-DAP” FOR GRAPHIC REPRESENTATION OF DIAMETER DISTRIBUTIONS}

\begin{abstract}
This research presents a new method of exploratory data analysis for diameter classes in natural tropical forests by using the graph denominated "UPLOT-DAP" used as support tool in the construction of frequency histograms. The research aimed to develop a new graphic solution, different from the traditional methods, for the determination of the diameter classes. To develop and to test the new graphic method were used 28320 DBH outside bark coming from 15 blocks of 96 hectares of the National Forest of Tapajós, Belterra, Pará, Brazil. The new method is quite flexible and essentially practical consisting in the use of visual analysis techniques of data as a way of diameters dispersion interpretation. The graphs were built considering a diameter class interval of $10 \mathrm{~cm}$ adopted as reference value. Two blocks were chosen with DBH $(\mathrm{cm})$ representative range of the population, block 18 [55,225] and block 25 [55,195] to discuss the results. The outcoming of the new method is to avoid the mistakes in constructing inexistent diameter profiles or different from the reality. The efficiency of the new method is based on its capacity to control the variation of the data so that the analysis are
\end{abstract}

\footnotetext{
1 Prof, Curso de Eng. Ftal da UFAM Doutorando em Manejo Ftal pela UFPR. uscfua@ig.com.br/ucunha@ fua.br. 92 647-4042/647-4043 Av. Gal. Rodrigo Otávio J. Ramos, 3000, Aleixo, Minicampus Universitário, CEP: 69077-000, Manaus-AM.

2 Prof Senior, Dr. em Manejo Ftal. Curso de Eng. Ftal da UFPR. PQ 1A - CNPq. sammac@ floresta.ufpr.br.

3 Prof. Senior, Dr. em Manejo Ftal. Curso de Eng. Ftal da UFPR. PQ 2A - CNPq. afig@ floresta.ufpr.br.

4 Prof. Senior, Dr. em Economia e Manejo Ftal. Curso de Eng. Ftal da UFPR. PQ 1B-CNPq. hosokawa@floresta.ufpr.br.
} 
more consistent with the physical and biological factors that characterize the dynamics of natural forests. The method also showed that the attempt of establishing a constant width or with a growing constant variation for the data, it can result in biased conclusions on the diameter structure of the forest.

Key-words: Diameter of the class, Graphic solution, Tropical Forest

\section{INTRODUÇÃO}

Através da distribuição diamétrica é possível descrever uma floresta ou os indivíduos de uma espécie em termos de abundância $(n / h a)$, dominância $\left(m^{2} / h a\right)$ ou freqüência $(\%)$. Há também a possibilidade de se conhecer as classes de diâmetro que apresentam maior número de indivíduos, área basal mais alta, ou maior volume, em cada espécie.

Nos histogramas para a construção gráfica utiliza-se uma tabela com classes de diâmetro e respectivas frequiências, com amplitudes de classe não necessariamente iguais (como é o caso apresentado neste contexto), formando retângulos contíguos, mutuamente exclusivos, de base igual ao tamanho da classe correspondente e altura proporcional à sua freqüência. No entanto, o mais adequado é construir histogramas onde as alturas de cada retângulo correspondam à chamada densidade de classe, que é obtida através do quociente entre a frequiência relativa de cada classe e a amplitude da classe. Isto faz com que o histograma construído tenha área unitária.

Geralmente, em problemas florestais, dispõe-se de duas alternativas para se fixar o número e a amplitude de classes diamétricas. Por exemplo, se os dados apresentam uma distribuição assimétrica próxima da normal, pode-se usar o intervalo de confiança dado por

$$
\begin{aligned}
& {\left[\bar{x}-t \times s_{\bar{x}} \leq x \leq \bar{x}+t \times s_{\bar{x}}\right]} \\
& \therefore s_{\bar{x}}=\frac{s_{x}}{\sqrt{n}} \text { sendo } x=D A P
\end{aligned}
$$

Neste caso, divide-se a amplitude total em 7 classes em função do desvio padrão $\left(\mathrm{s}_{\mathrm{x}}\right)$, sendo usual a seguinte abordagem: $\bar{x}-3 s_{x}, \bar{x}-2 s_{x}, \bar{x}-s_{x}, \overline{\mathrm{x}}, \bar{x}+s_{x}, \bar{x}+2 s_{x}, \bar{x}+3 s_{x}$. Se, ao contrário, os dados apresentarem uma distribuição decrescente do tipo exponencial negativa, pode-se fixar uma amplitude arbitrária para todas as classes, ex. $5,8,10,12 \mathrm{~cm}$ com base em recomendações de estudos já realizados. Esse procedimento tem a desvantagem de não permitir a caracterização adequada das classes extremas, principalmente quando os dados são coletados a partir de um diâmetro mínimo estabelecido que varia com os objetivos, mas que pode ser de $10 \mathrm{~cm}$ para regeneração natural e a partir de $30 \mathrm{~cm}$, sendo mais comum 35, 45 ou $55 \mathrm{~cm}$ nos inventários comerciais da Amazônia.

O método gráfico desenvolvido denomina-se “UPLOT-DAP"e tem por finalidade auxiliar o pesquisador na preparação dos seus dados para estudos que requeiram interpretação visual ou análises sobre o comportamento de variáveis por classes diamétricas.

O “UPLOT-DAP”é uma construção gráfica com suporte analítico que visa servir de alternativa complementar à análise tradicional de dados com respeito às classes diamétricas. Permite definir classes diamétricas por meio de representação gráfica que é muito mais clara do que as construções habituais. Não tem a finalidade de substituir o histograma, mas de servir de apoio na sua construção, de modo que, combinando-se essas duas formas de representação gráfica, consiga-se melhores critérios de definição das amplitudes e números de classes diamétricas.

O "UPLOT-DAP" difere do tipo DOT PLOT (gráfico de pontos), onde os dados de um conjunto de observações são todos representados explicitamente sobre uma reta e cada ponto do gráfico representa um determinado número de repetições. Embora a forma gráfica apresente algumas semelhanças, os dois tipos possuem propriedades distintas.

A pesquisa objetivou desenvolver uma nova solução gráfica, diferente dos métodos tradicionais, para a determinação das classes diamétricas. 


\section{MATERIAIS E MÉTODOS}

\section{Base de dados}

A área utilizada (fig. 1) tem formato retangular $4 \mathrm{~km}$ x 3,6 km (1440 ha), compõese de 15 blocos contíguos de 96 ha de floresta primária, cada um medindo $800 \mathrm{~m}$ no sentido leste-oeste e $1200 \mathrm{~m}$ no sentido norte-sul e faz parte da Floresta Nacional do Tapajós (FLONA TAPAJÓS).

Esta FLONA tem 600.000 ha e está situada entre os paralelos de $2^{\circ} 40^{\prime}$ a $4^{\circ} 10^{\prime}$ de latitude sul e os meridianos de $54^{\circ} 45^{\prime}$ a $55^{\circ}$ 30' de longitude oeste de Greenwich, município de Belterra, Pará. O seu manejo está previsto para 2001, como parte do programa a ser executado pela TREVISO AGROPECUÁRIA LTDA, através de Convênio de Cooperação entre o Instituto Brasileiro do Meio Ambiente e dos Recursos Naturais Renováveis (IBAMA) e a Internacional Tropical Timber Organization (ITTO), em andamento desde 1999.

De acordo com Radambrasil (1976), predominam na área, latossolos amarelos distróficos com diferentes texturas, profundos, ácidos, friáveis, onde ocorre a floresta densa. Como variação, tem-se também o latossolo amarelo distrófico plíntico de textura média e argilosa, os solos concrecionários lateríticos indiscriminados distróficos e areias quartzosas distróficas. Estes solos ocorrem em diversos tipos de relevo, variando desde plano a forte ondulado, sob vegetação de floresta densa e são originados a partir de sedimentos do Terciário.

A Floresta Ombrófila Densa subdividida pelos quadrantes ecológicos (litologia, morfologia e clima), em termos de aspectos fisionômicos e florísticos, caracteriza a tipologia florestal dominante na região. Apresenta-se também na área uma fisionomia florestal densa, com indivíduos emergentes, ocupando as áreas aplainadas dos platôs do Xingu-Tapajós, caracterizada não só por apresentar alto volume de madeira, como também pela presença de espécies de alta cotação de preços nos mercados externo e interno (IBAMA/PNUD, 1997).

Os dados provêm do inventário florestal a $100 \%$ realizado pela Fundação de Pesquisas Florestais do Paraná (FUPEF) no ano de 1984, mediante convênio firmado com o então Instituto Brasileiro de Desenvolvimento Florestal (IBDF). Foram utilizados os DAP $\geq 55 \mathrm{~cm}$ com casca de 28.320 árvores, provenientes dos 15 blocos contíguos de floresta primária, conforme mencionado anteriormente.

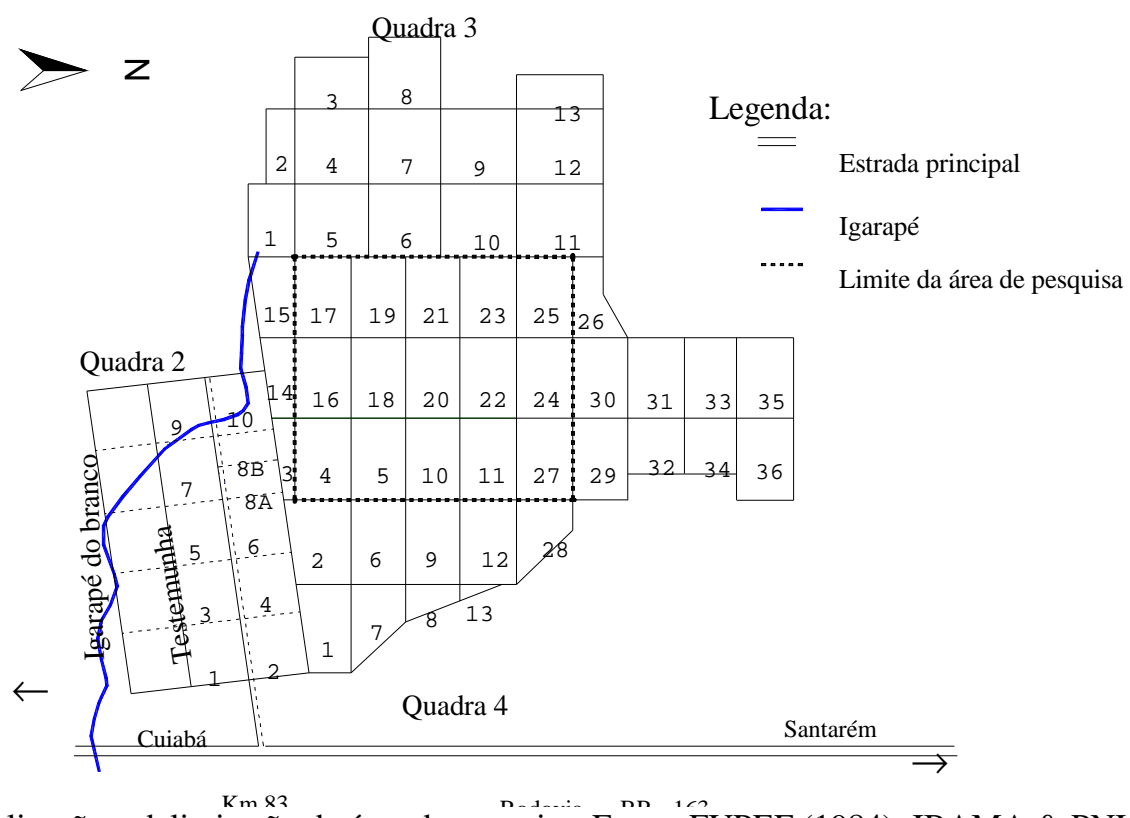

Figura 1: Localização e delimitação da área de pesquisa. Fonte: FUPEF (1984); IBAMA \& PNUD (1997) Figure 1: Location and delimitation of the research area. Source: FUPEF (1984); IBAMA \& PNUD (1997) 


\section{Princípios Da Construção Gráfica}

Nas construções gráficas desta pesquisa, procurou-se também o apoio da Neográfica (La Graphique), disciplina sistematizada por Jacques Bertin (Laboratoire de Graphique, École des Hautes Études en Sciences Sociales, Paris), que propõe princípios que permitem transformar um gráfico ilustração numa construção útil ao pesquisador. Nesse processo, simplificar os dados é excelente técnica para evidenciar as relações entre eles, pois a simplificação tem por objeto fazer aparecer as relações, ou seja, a informação pesquisada (Bertin, 1986). Seguidor de Bertin, Bonin (1983) menciona que a escolha de classes numa série estatística é um problema de simplificação dos dados em função de uma escolha racional e enfatiza que na representação eficaz de uma série estatística, supõe-se que a estrutura da série seja respeitada.

De acordo com Prodan (1968), a representação gráfica da distribuição diamétrica tem a finalidade de facilitar a percepção e a compreensão de uma informação numérica. Um gráfico é capaz de ilustrar de maneira simples o significado dos dados, pois de outro modo exigiria mais tempo, trabalho e habilidade para interpretá-los.

Para Tufte (2001) modernos gráficos de dados podem fazer mais do que simplesmente substituir pequenas tabelas estatísticas. Na sua melhor forma, gráficos são instrumentos para raciocinar sobre informação quantitativa. Frequientemente o modo mais efetivo para descrever, explorar e sumarizar um conjunto de números, até mesmo um grande conjunto, é observar as figuras destes números. Além disso, de todos os métodos para analisar e comunicar informação estatística, gráficos de dados bem construídos são usualmente os mais simples e ao mesmo tempo os mais poderosos.

Excelência em gráficos estatísticos consiste de idéias complexas comunicadas com clareza, precisão e eficiência. De acordo com Tufte (2001) uma construção gráfica deveria:

i) Mostrar os dados,

ii) Induzir o observador preferivelmente a pensar sobre a substância antes de pensar sobre a metodologia, desenho gráfico, a tecnologia de produção gráfica ou algo semelhante,

iii) Evitar distorcer o que os dados têm a dizer,

iv) Apresentar muitos números em um pequeno espaço,

v) Tornar grandes conjuntos de dados coerentes,

vi) Encorajar o olho a comparar diferentes partes dos dados,

vii) Revelar os dados em vários níveis de detalhes a partir de uma avaliação ampla da sua estrutura,

viii) Servir a um propósito razoavelmente claro: descrição, exploração, tabulação ou decoração.

Para Löetsch et al. (1973), se as árvores de um povoamento forem agrupadas em certos intervalos de diâmetros à altura do peito, obtém-se a distribuição diamétrica. Além das frequiências, também a área basal, volume ou incrementos em área basal podem ser plotados em classes diamétricas.

De acordo com Löetsch et al. (1973), as distribuições decrescentes são importantes porque ocorrem em alguns tipos de florestas naturais com alta diversidade de espécies e variação de idades. As distribuições decrescentes ocorrem principalmente em:
a) florestas naturais;
b) plantios florestais manejados com uma espécie em várias idades;
c) plantios florestais manejados com várias espécies e idades.

A distribuição diamétrica decrescente pode ser de 3 tipos, em função do decréscimo do número de árvores em classes sucessivas de diâmetro:

Tipo I - O número de árvores por unidade de área decresce nas classes sucessivas de diâmetros em uma progressão geométrica uniforme,

$$
n_{1}=q n_{2}=q^{2} n_{3}=\mathrm{L}=q^{k-1} n_{k}
$$

Tipo II - O número de árvores por unidade de área decresce nas classes sucessivas de diâmetro em uma progressão geométrica crescente, 


$$
n_{1}>q n_{2}>q^{2} n_{3}>\mathrm{L}>q^{k-1} n_{k}
$$

Tipo III - O número de árvores por unidade de área decresce nas classes sucessivas de diâmetro em uma progressão geométrica decrescente.

$n_{1}<q n_{2}<q^{2} n_{3}<\mathrm{L}<q^{k-1} n_{k}$

sendo $q=\frac{n_{1}}{n_{2}}>\frac{n_{2}}{n_{3}}>\mathrm{L}>\frac{n_{k-1}}{n_{k}}=$ quociente de De

Liocourt

em que

$n_{i}=$ número de árvores por classe de DAP (i=

$1,2, \ldots, \mathrm{k})$ e por unidade de área

$q=$ fator correspondente à mudança entre classes sucessivas

A construção de intervalos de classes pode ser feita pela amplitude total $\left(w=d_{+}-d_{0}\right), \quad$ dividindo-se $w$ por um determinado tamanho e tabulando as freqüências associadas com cada intervalo. $\mathrm{O}$ número de intervalos de classes depende do número de observações. Quanto maior o número de observações, mais intervalos de classes são requeridos. Segundo Hoshmand (1998), geralmente usa-se entre 5 a 15 intervalos de classe. Pode-se ainda usar a fórmula (05), sugerida por Sturges (1926) para se determinar o número de intervalos de classe.

$k=1+3,322 \times \log _{10} n$

em que

$\mathrm{k}=$ número de intervalos de classe

$n=$ número de observações

De acordo com Löetsch et al. (1973), várias comparações exigem que as amplitudes de classes sejam iguais e os limites de classes coincidentes. Estas condições são satisfeitas quando um mesmo objeto está sendo investigado. Para comparações de diferentes distribuições, as amplitudes de classes podem ser iguais ou diferentes, porém diferindo nos limites de classes. Transformações das frequiências para intervalos de classes comparáveis podem ser obtidas através da curva de frequiência cumulativa. Deve-se comparar as distribuições não somente pelas quantidades absolutas, mas pelas relações entre as classes específicas de interesse. Freqüências comparáveis podem ser obtidas de várias maneiras:

i) Pelo uso de valores índices, ou seja, número de troncos por unidade de área, onde se elimina a diferença resultante das variações das populações por áreas;

ii) Usando freqüências relativas para comparação, o que permite melhor reconhecimento das características comuns das distribuições diamétricas de freqüências de diferentes variáveis;

iii) Pela formulação matemática da distribuição presente dos diâmetros através de funções com distribuição flexível (objeto da presente pesquisa).

Testou-se o uso de valores aleatórios procedentes da distribuição uniforme $[0,1]$ e distribuição Beta $[\alpha, \beta]$ no cálculo da dispersão do diâmetro por permitirem que os valores da variável aleatória DAP se distribuam como eventos ou probabilidades mesmo em caso de distribuição truncada.

Para uma variável aleatória $X=x$, distribuída uniformemente sobre o intervalo $[a, b]$, onde $a<b$, tem-se que sua função densidade de probabilidade $f(x)$ é dada por:

$$
f(x)\left\{\begin{array}{lrc}
=\frac{1}{b-a} & \text { para } & a \leq x \leq b \\
=0 & & \text { em outras circunstâncias }
\end{array}\right.
$$

Uma variável aleatória $\mathrm{X}=\mathrm{x}$ tem uma distribuição Beta com parâmetros $\alpha, \beta$ se sua função densidade de probabilidade $[\mathrm{f}(\mathrm{x})]$ for dada por :

$$
\begin{gathered}
f(x)=\frac{\Gamma(\alpha+\beta)}{\Gamma(\alpha) \Gamma(\beta)} x^{\alpha-1}(1-x)^{\beta-1} \quad \text { para } \\
0<x<1
\end{gathered}
$$

em que

$f(x)=$ função densidade de probabilidade

$\alpha, \beta=$ parâmetros da função Beta 
$\Gamma(\cdot)=$ função

gama,

$\Gamma(\alpha)=\int_{0}^{\infty} y^{\alpha-1} e^{-y} d y$

para

$\alpha>0$

$x=$ DAP transformado pela equação (08)

Obviamente que o emprego da função Beta expressa pela equação (2) no ajuste de distribuições diamétricas, exige que sejam feitas transformações nos dados que atendam ao campo de domínio da função. Como quase sempre acontece, os DAP medidos nos inventários começam a partir de valores como $10,20,30,35,45$ e $55 \mathrm{~cm}$, originando uma distribuição truncada. A principal vantagem no uso da função Beta está na facilidade de ajuste e no fato de que o ajuste para dados truncados não exige a recuperação dos parâmetros da distribuição uma vez que a transformação indicada na equação (08) envolvendo o DAP, executa uma translação de eixo que torna os limites dos dados compatíveis com o domínio da função.

$\mathrm{x}=\left(\mathrm{d}+0,1-\mathrm{d}_{0}\right) /\left(\mathrm{d}_{+}+0,2-\mathrm{d}_{0}\right)$

em que

$$
\begin{aligned}
& \mathrm{X}=\text { valor adimensional } \\
& d=\text { diâmetro a altura do peito (DAP), em } \mathrm{cm} \\
& \mathrm{d}_{0}=\text { menor DAP observado, em } \mathrm{cm} \\
& \mathrm{d}_{+}=\text {maior DAP observado, em } \mathrm{cm}
\end{aligned}
$$

\section{Princípios de Construção do "UPLOT- DAP"}

Para o conjunto de DAP's de cada bloco, foram geradas duas funções visando à construção gráfica. A primeira, $f(x)$, proporcionada pelo ajuste da distribuição Beta para os DAP observados. A segunda, $f^{\prime}(x)$, resultante do produto da multiplicação de $f(x)$, função densidade de probabilidade da distribuição Beta, ou pelos números aleatórios da distribuição uniforme $\{@$ rand $[0,1]\}$ ou pelos números aleatórios da própria distribuição Beta $\{@$ rand $[\alpha, \beta]\}, \quad$ gerados usando-se os parâmetros estimados $\alpha, \beta$.

A seguir, plotou-se cada par de dados $\left[d, f^{\prime}(x)\right]$, em modo de dispersão, o que resultou nos gráficos "UPLOT-DAP", conforme pode ser observado nas Figuras 2 e 4. Havendo necessidade de realçar a escala e melhorar a visualização dos pontos no gráfico, pode-se adicionar $\sqrt{\alpha}$ ao cálculo de $f^{\prime}(x)$ resultando na seguinte expressão: $f^{\prime}(x)+\sqrt{\alpha}$. $\mathrm{O}$ método deve seguir as seguintes fases e passos:

\section{Fase de Preparação dos Dados}

Passo 1: Utilizar a equação (08) para transformar a coleção de DAP (cm), visando o ajuste da distribuição Beta.

Passo 2:. Ajustar a distribuição Beta para a obtenção dos parâmetros $\alpha$ e $\beta$.

Passo 3. Utilizar os parâmetros $\alpha$ e $\beta$ para obter a geração de números aleatórios através da função Beta e para calcular a função densidade de probabilidade $f(x)$. Em seguida, multiplicar $\mathrm{f}(\mathrm{x})$ por @ rand $[\alpha, \beta]$ ou por @ rand $[0,1]$, obtendose $f^{\prime}(x)$. Portanto, observa-se que $f^{\prime}(x)$ difere de $f(x)$ apenas em termos do mecanismo aleatório responsável pela realização dos valores de $f^{\prime}(x)$.

\section{Fase de Construção}

Passo 1: Plotar os pares [DAP, $\left.\mathrm{f}^{\prime}(\mathrm{x})\right] \mathrm{em}$ modo de dispersão ${ }^{x y}$, para obter o gráfico “UPLOT-DAP”.

Passo 2: Construir um histograma-base utilizando uma amplitude de referência com valores constantes de 5 ou $10 \mathrm{~cm}$. Recomenda-se usar a amplitude $5 \mathrm{~cm}$ para análises ao nível de espécies e $10 \mathrm{~cm}$ para a floresta como um todo.

Passo 3: Movimentar as classes de DAP (cm) de modo que o perfil observado no "UPLOT-DAP" seja transcrito para o Histograma, conservando o máximo grau de semelhança com a forma explícita da distribuição dos pontos do "UPLOTDAP". A movimentação das classes de DAP (cm) significa fusão de classes adjacentes subsequientes com redimensionamento das amplitudes de classes ou até mesmo a manutenção da 
estrutura de classes desde que venham a ser consideradas uma característica típica da espécie ou da floresta.

Fase Final: Para que as alterações no histograma sejam consideradas satisfatórias é necessário que o perfil do histograma seja idêntico ao "UPLOTDAP" o que caracterizará o fim do processo, obtendo-se a distribuição das classes diamétricas construída com base em princípios gráficos.

\section{Análise Estatística}

Após a construção gráfica realizou-se a análise estatística abordando os seguintes aspectos: medidas de tendência central e de variabilidade para dados agrupados como média, mediana e desvio-padrão. Para maiores detalhes sugere-se consultar (Costa Neto, 1999 ; Moore, 2000).

\section{Quantificação do Erro para Dados em Classes}

Conforme Husch et al. (1982), classes para variáveis contínuas são freqüentemente estabelecidas a fim de facilitar o manuseio de dados nas computações. Freqüências podem então ser assinaladas para estas classes. Estas frequiências representam a ocorrência ou recorrência de certas medidas de uma variável contínua que teve de ser organizada em grupo ou classe de limites definidos por razão de conveniência. Sendo que, os intervalos de classes deveriam ser escolhidos de maneira que seus centros de classe coincidam com dados observados. Esse procedimento tende a diminuir o erro de agrupamento nas análises estatísticas.

A análise teórica do efeito do cálculo das estimativas para freqüências agrupadas é feita sob a hipótese de que as observações ocorrem em uma distribuição regular assumindo-se um acréscimo ou decréscimo linear das freqüências dentro de cada classe. Quando as observações são agrupadas em classes, o centro de classe representa a média que será usada em outros cálculos. A média verdadeira, entretanto, pode desviar-se do centro de classe, aleatoriamente ou sistematicamente.

De acordo com Prodan (1968), o agrupamento de observações em classes com intervalos constantes e os cálculos dos valores médios das classes resulta em dois tipos de erros, um sistemático e o outro aleatório, que podem ser examinados com a ajuda da distribuição unimodal e distribuição decrescente.

\section{Erro Sistemático}

De acordo com Löetsch et al. (1973, p. 85), o erro sistemático é causado porque o diâmetro médio de todas as árvores da classe $\mathrm{i}$ é assumido como sendo o diâmetro centro de classe $d_{i}$, o qual não coincide nem com o diâmetro médio $\bar{d}_{i}$ nem com o diâmetro da área transversal média da classe $d_{g i}$.

O diâmetro da área tranversal média da classe $i, d_{g i}$, é sempre maior do que o diâmetro médio aritmético $\bar{d}_{i}$. Nestas condições, a área basal da classe $i$ será

$$
G_{i}^{\prime}=\frac{\pi}{4} n_{i} d_{i}^{2}
$$

em que

$$
\begin{aligned}
& \mathrm{n}_{\mathrm{i}}=\text { número de árvores na classe } i \\
& d_{i}=\text { diâmetro centro de classe } i
\end{aligned}
$$

Como esse valor é afetado pelo erro, a área basal verdadeira da classe $\mathrm{i}$ é obtida por

$$
\begin{aligned}
G_{i} & =\sum_{j=1}^{n_{i}} g_{i j}=\frac{\pi}{4} \sum_{j=1}^{n_{i}} d_{i j}^{2}=\frac{\pi}{4} n_{i} d_{g i}^{2} \\
d_{g i} & =\sqrt{\frac{\sum_{j=1}^{n_{i}} d_{i j}^{2}}{n_{i}}}
\end{aligned}
$$

em que

$$
g_{i j}=\text { área tranversal da árvore } \mathrm{j} \mathrm{da}
$$
classe $i$

$d_{i j}=$ diâmetro da árvore $\mathrm{j}$ da classe $i$

$d_{g i}=$ diâmetro correspondente à área transversal média da classe $i$

O cálculo do erro sistemático absoluto da classe $i\left(E_{s i}\right)$ devido ao agrupamento resulta então

$$
E_{s i}=G_{i}^{\prime}-G_{i}=\frac{\pi}{4} n_{i}\left(d_{i}^{2}-d_{g i}^{2}\right)
$$


Assim, a diferença entre o diâmetro centro de classe $\left(d_{i}\right)$ e o diâmetro da área transversal média $\left(d_{g i}\right)$ é decisivo para a magnitude do erro sistemático da área basal, devido ao agrupamento em classes. Essa diferença pode ser estimada em duas fases:

Fase 1. Determinação da diferença $\left(A_{i}\right)$ entre o diâmetro centro de classe ${ }^{\left(d_{i}\right)}$ e o diâmetro médio aritmético $\left(\bar{d}_{i}\right)$ da classe $i$

$$
A_{i}=d_{i}-\bar{d}_{i}
$$

Fase 2. Determinação da diferença $\left(A_{i}^{\prime}\right)$ entre a média aritmética do diâmetro da classe $\left(\bar{d}_{i}\right)$ e o diâmetro da classe de área basal média $\left(d_{g i}\right)$

$$
A_{i}^{\prime}=\bar{d}_{i}-d_{g i}
$$

Assumindo-se uma área basal $\mathrm{G}_{\mathrm{i}}$ sem erro, o cálculo do erro sistemático $\left(\mathrm{E}_{\mathrm{s}}\right)$ em $\%$ é obtido por

$$
E_{s} \%=\frac{d_{i}^{2}-d_{g i}^{2}}{d_{g i}^{2}} \times 100
$$

Assumindo-se que a área transversal média $\left(d_{g i}\right)$ é afetada por erro, tem-se que

$$
E_{s}^{\prime} \%=\frac{d_{i}^{2}-d_{g i}^{2}}{d_{i}^{2}} \times 100
$$

Um fator $\mathrm{k}_{\mathrm{i}}$ para ajustar o erro da área basal da classe i é obtido por

$$
k_{i}=\frac{d_{g i}^{2}}{d_{i}^{2}}=\frac{\bar{d}_{i}^{2}+\frac{w_{i}^{2}}{12}}{d_{i}^{2}}
$$

em que $w_{i}=$ amplitude da classe $i$

Pode-se afirmar que o erro sistemático devido ao agrupamento em classes é particularmente severo em intervalos de classes com amplitudes maiores e nas amplitudes de distribuições com fortes aumentos ou declínios.

\section{Erro Aleatório}

Para Löetsch et al. (1973), este tipo de erro por sua natureza é atribuído à representação insuficiente da distribuição presente das árvores medidas com suta. Em amostras com pequeno número de indivíduos onde a distribuição de uma pequena parte da população é usada para obter conclusões sobre a população total - com um número variável de diâmetros por classe, esse erro pode exceder consideravelmente, uma vez que a magnitude do erro aleatório diminui com o aumento do número de DAP's medidos.

A variância dos valores do diâmetro em torno do centro de classe de uma distribuição próxima da retangular é obtida por:

$$
s_{d i}^{2}=\frac{w_{i}{ }^{2}}{12} \quad \therefore \quad s_{d i}=\sqrt{\frac{w_{i}{ }^{2}}{12}}=\frac{w_{i}}{2 \sqrt{3}} \cong 0,3 w_{i}
$$

O erro padrão do diâmetro médio aritmético da classe i é então obtido por:

$$
S_{\overline{d i}}= \pm \frac{0,3 w_{i}}{\sqrt{n_{i}}}
$$

em que $\mathrm{n}_{\mathrm{i}}=$ número de árvores medidas em cada classe $\mathrm{i}$.

Por meio da fórmula (18), válida para uma distribuição retangular é possível estimar também o erro padrão de classes de distribuições com amplitudes crescentes ou decrescentes.

$$
\text { A influência do erro aleatório (Ea\%) }
$$
do agrupamento em classes sobre a área basal pode ser estimado a partir de:

$$
E_{a} \% \cong \pm \frac{60 w_{i}}{d_{i} \sqrt{n_{i}}}
$$

Utilizou-se os gráficos em separado para estruturar os histogramas de frequiência, variando a amplitude de classe em função da dispersão dos pontos exibidos no "UPLOTDAP". Utilizou-se a amplitude de $10 \mathrm{~cm}$ para servir de base na definição a priori dos intervalos de classes por duas razões: primeira, 
por sua praticidade e pelo uso já consagrado dessa medida e também por ser um produto de pesquisas realizadas sobre distribuições diamétricas na Amazônia (vide Cunha (1995), Barros (1980)).

Para expressar os pontos no "UPLOTDAP" deve-se recorrer a um gráfico de dispersão $x, y$ para plotar os pares de coordenadas geradas e visualizar os dados num plano de observação dominados por pontos que se comportam como coordenadas vetoriais em sua respectiva classe diamétrica.

\section{RESULTADOS E DISCUSSÃO}

Considerando que a aplicação do método para os 15 blocos apresentou resultados similares, decidiu-se escolher os blocos 18 [55, 225] e 25 [55,195] para demonstrar as técnicas de análise, visto que representam bem a variação total dos dados analisados.

A tabela 1 mostra que no bloco18, o erro aleatório $E_{a} \%$ devido ao agrupamento foi inferior a $1 \%$ somente até a $5^{\text {a }}$ classe $[95,105]$. Verifica-se que o erro sistemático $E_{s} \%$ no bloco 18 , variou de 0,59 na $4^{\mathrm{a}}$ classe $[85,95]$ a $9,53 \%$ na $6^{\mathrm{a}}$ classe $[105,125]$. O quociente de

De Liocourt $\mathrm{q}_{\mathrm{i}}$ se manteve com a mesma tendência para o bloco 18. Apesar do erro sistemático ter apresentado em alguns casos, valores superiores à precisão científica recomendada que, segundo Prodan (1968), deve ser de 1 a $2 \%$, fez-se necessário aplicar o fator de correção $k_{i}$ dado pela equação (16), para melhorar a precisão das estimativas.

Tabela 1: Estimativa do erro sistemático $\left(E_{s} \%\right)$, erro aleatório $\left(E_{a} \%\right)$ e quociente de De Liocourt $\left(q_{i}\right)$ dos blocos 18 e 25

Table 1: Estimate of the systematic error $\left(E_{s} \%\right)$, random error $\left(E_{a} \%\right)$ and quotient of De Liocourt $\left(q_{i}\right)$ of the blocks 18 and 25

\begin{tabular}{llllllll}
\hline Bloco 18 & $E_{s} \%$ & $E_{a} \%$ & $q_{i}$ & $\begin{array}{c}\text { Bloco } 25 \\
\text { DAP }(\mathrm{cm})\end{array}$ & $E_{s} \%$ & $E_{a} \%$ & $q_{i}$ \\
DAP $(\mathrm{cm})$ & 1,83 & 0,39 & 1,5 & $55 \delta 65$ & 0,63 & 0,37 & 1,7 \\
$55 \delta 65$ & 0,91 & 0,41 & 1,3 & $65 \delta 75$ & 1,27 & 0,41 & 1,7 \\
$65 \delta 75$ & 1,06 & 0,40 & 1,9 & $75 \delta 85$ & 0,32 & 0,46 & 1,7 \\
$75 \delta 85$ & 0,59 & 0,49 & 1,6 & $85 \delta 95$ & 0,19 & 0,53 & 1,2 \\
$85 \delta 95$ & 5,67 & 0,56 & 1,5 & $95 \delta 105$ & 3,68 & 0,52 & 2,4 \\
$95 \delta 105$ & 9,53 & 1,19 & 3,0 & $105 \delta 125$ & 3,68 & 1,38 & 2,7 \\
$105 \delta 125$ & 0,95 & 1,74 & 2,6 & $\geq 125$ & 7,59 & 5,73 & \\
$125 \delta 145$ & $-0,54$ & 8,20 & & & & & \\
$\geq 145$ & & & & & & &
\end{tabular}

Ainda de acordo com a Tabela 1 no bloco 25 o $E_{s} \%$ apresentou uma variação de 0,32 na $3^{\mathrm{a}}$ classe $[75,85]$ a $7,59 \%$ na $7^{\mathrm{a}}$ classe $[\geq 125]$. O $E_{a} \%$ manteve-se inferior a $1 \%$ até a $5^{\text {a }}$ classe do bloco 25 [95,105], alcançando maiores índices na $7^{\mathrm{a}}$ classe $[\geq 125] \mathrm{com}$ $5,73 \%$. O $q_{i}$ manteve-se constante nas 3 primeiras classes do bloco 25 e crescente nas demais classes. De um modo geral, nesse bloco, os níveis de erro aleatório são considerados satisfatórios ( $1 \%$ a $2 \%$ ) em todas as classes, excetuando-se a última classe. Nos casos em que houver discrepância no erro sistemático, deve-se recorrer ao fator de correção $k_{i}$ para melhorar a precisão das estimativas. 


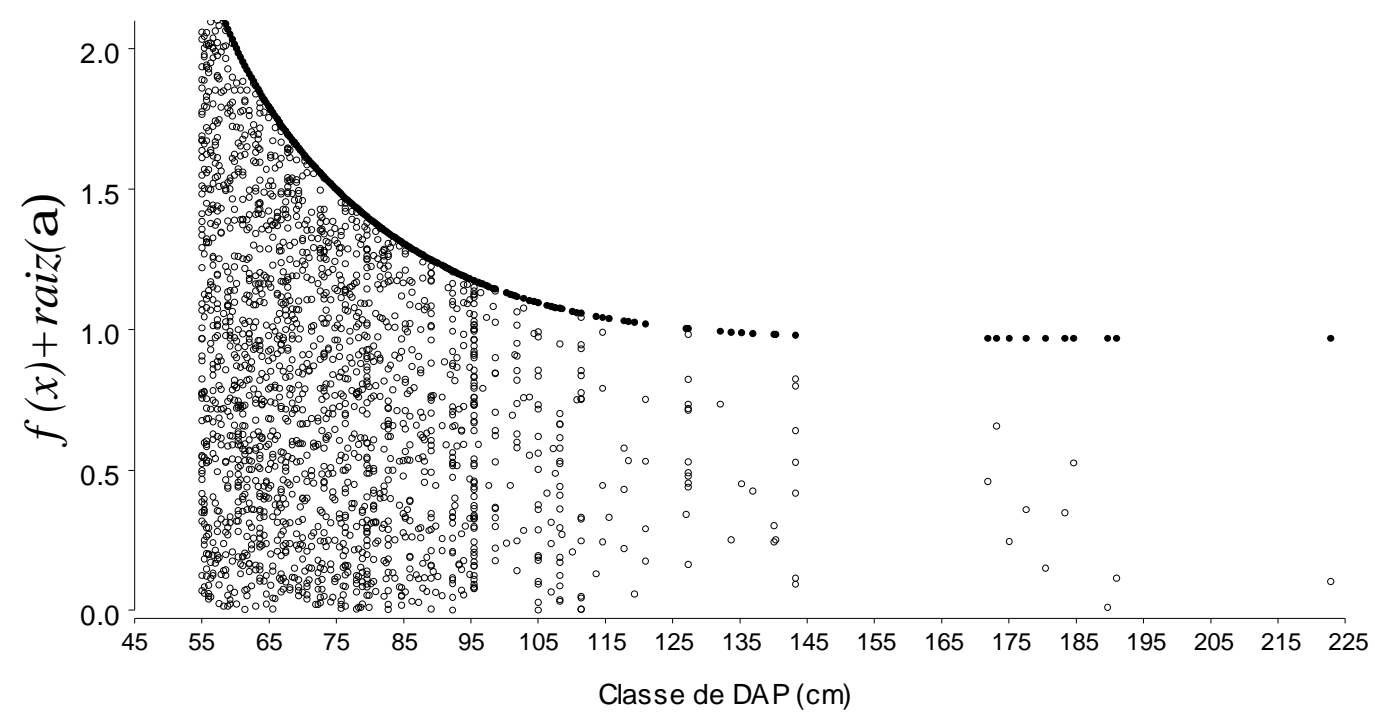

Figura 2: “UPLOT-DAP”do bloco 18.

Figure 2: “UPLOT-DAP” of the block 18.

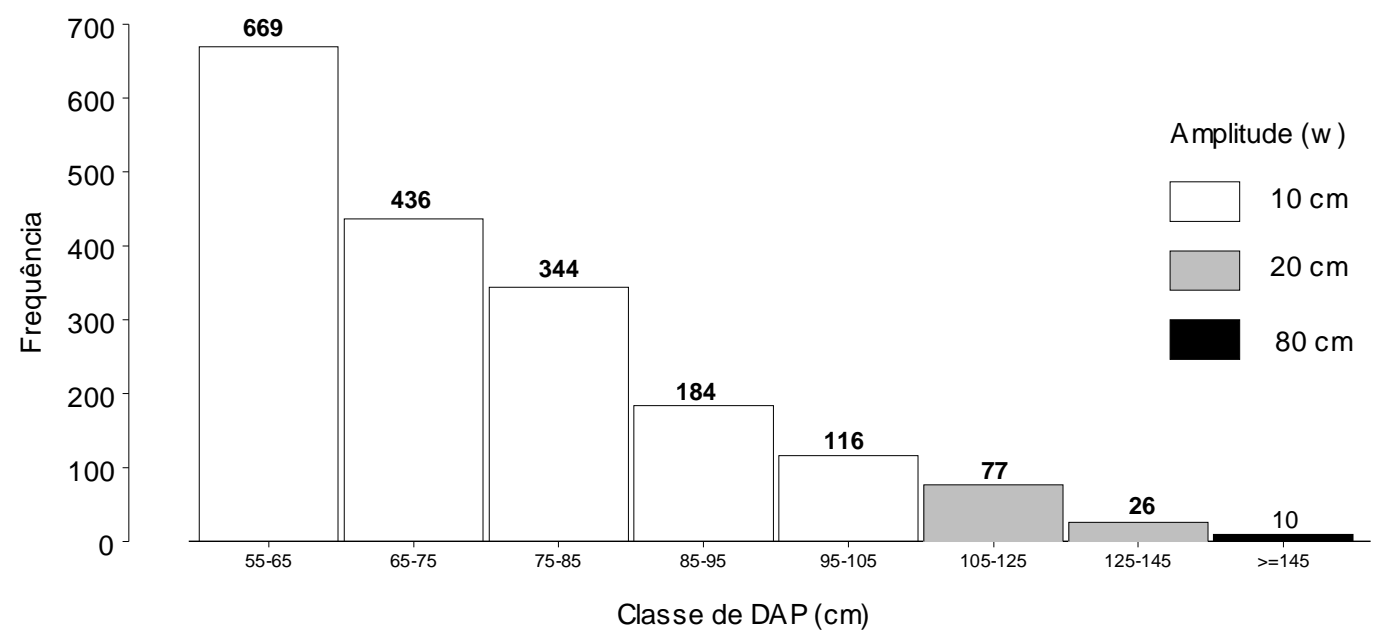

Figura 3: Histograma do bloco 18 construído a partir do "UPLOT-DAP"da figura 2

Figure 3: Histogram of the block 18 built based on the "UPLOT-DAP" of the figure 2

Observando-se a figura 3 , tem-se um histograma com 8 classes, construído interativamente com base na figura 2 e que até a $5^{\text {a }}$ classe [95-105], as frequiências representaram bem a relação entre os dados, mantendo a mesma amplitude de $10 \mathrm{~cm}$. Na $6^{\text {a }}$ classe [105-125] e na $7^{\mathrm{a}}$ classe [125-145], a amplitude foi de $20 \mathrm{~cm}$, pois de outra forma seria quebrada a tendência da variabilidade diamétrica em termos de freqüência das classes menores para as classes maiores. 


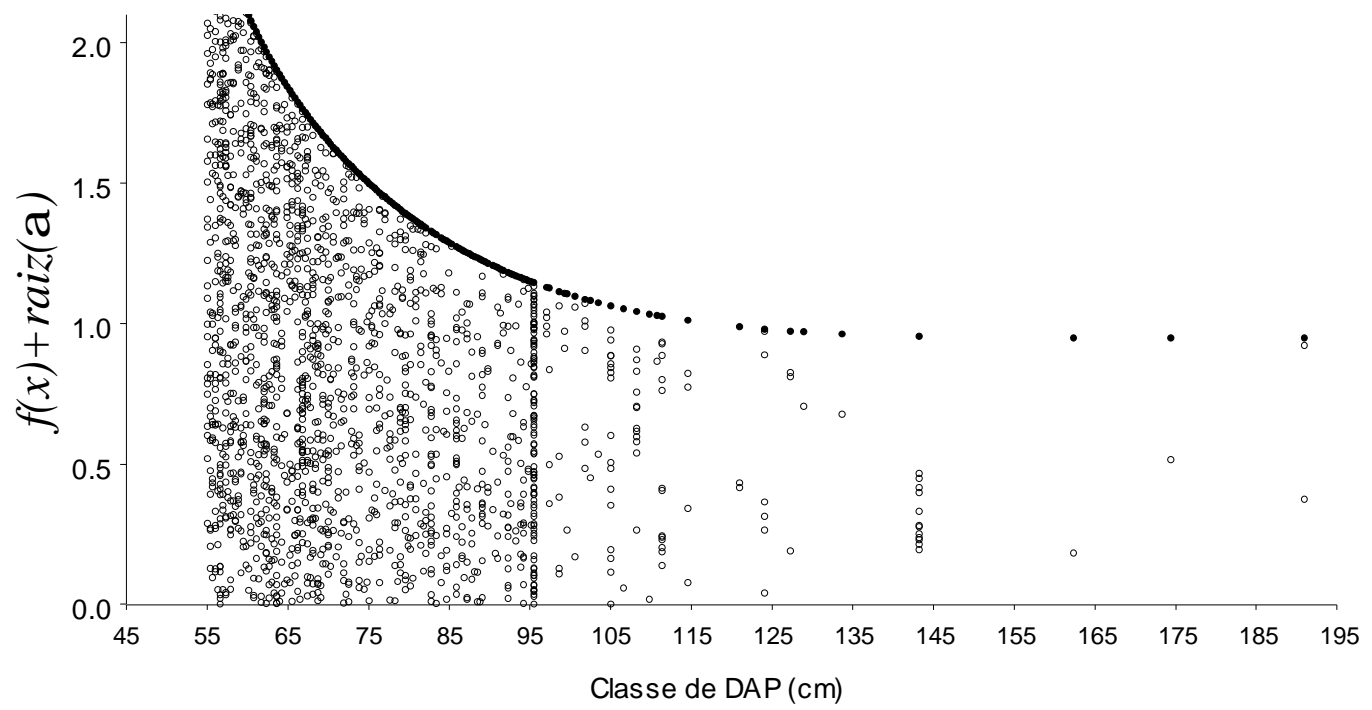

Figura 4: “UPLOT-DAP”do bloco 25

Figure 4: “UPLOT-DAP”of the block 25

Na última classe [ $>=145]$ a amplitude diferiu das demais e se fixou em $80 \mathrm{~cm}$ para permitir que a razão entre classes subseqüientes se mantivesse dentro dos princípios do quociente de De Liocourt para florestas naturais.

$\mathrm{Na}$ figura 5, apesar de se ter uma amplitude total [55,195] cerca de $30 \mathrm{~cm}$ menor, comparada ao bloco 18 , o histograma resultante da observação visual dos dados na Figura 4, se fixou em 7 classes. É fácil perceber que até a $5^{\mathrm{a}}$ classe [95-105] a amplitude manteve a constante de $10 \mathrm{~cm}$ e que na $6^{\mathbf{a}}$ classe $[105,125]$, ocorreu uma variação elevando essa amplitude para $20 \mathrm{~cm}$. Na última classe $\quad[>=125]$ a variação foi consideravelmente maior com a amplitude fixando-se em $70 \mathrm{~cm}$. Nesse caso, a maior contribuição do novo método foi o de respeitar as propriedades das classes que por razões biológicas apresentam vazios intermitentes ou detectam a presença de pontos raros esparsamente distribuídos (difusos). Uma solução diferente da apresentada certamente poderia trazer distorções na análise final dos dados.

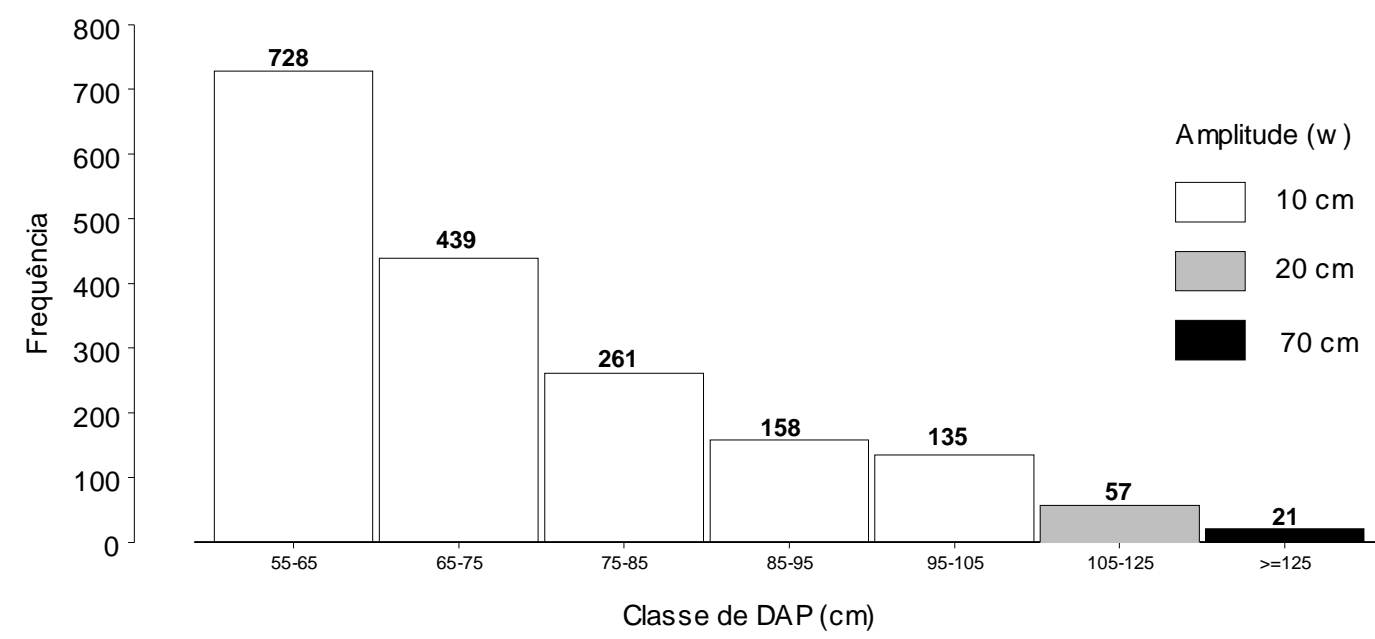

Figura 5: Histograma do bloco 25 construído a partir do "UPLOT-DAP”da figura 3

Figure 5: Histogram of the block 25 built based on the "UPLOT-DAP" of the figure 3 
A última classe [ $>=125]$ do bloco 25 , construída sob uma amplitude de $70 \mathrm{~cm}$, comportou-se de acordo com a teoria da distribuição $\mathbf{J}$ invertido para florestas naturais, fechando o ciclo de análise e quebrando alguns pressupostos teóricos, como sugerido por Hoshmand (1998), segundo o qual, o número de intervalos de classes depende do número de observações e quanto maior o número de observações, mais intervalos de classes são requeridos, ou seja, o número de classes tende a um limite com o aumento do número de observações.

O papel do novo gráfico em todos os casos analisados foi o de evitar a construção de um perfil diamétrico que não existe, pois, visualizando-se as classes diamétricas no "UPLOT-DAP" é possível perceber que enquanto em certas classes a nuvem de pontos varia entre densa, difusa ou uniforme, o mesmo não acontece com alguns segmentos de classes onde a presença rara ou a ausência de dados são eventos fáceis de serem identificados.

O fenômeno dos eventos raros e nulos repetiu-se em todos os casos analisados, podendo-se reafirmar o princípio de que a amplitude de classe é inversamente proporcional à freqüência local ou zonal, dependendo de como os dados estão dispersos ao longo do eixo que contém as classes diamétricas.

Foi observado que o "UPLOT-DAP" tem a vantagem de ser útil na re-expressão dos eventos em cada classe, pois neste tipo de floresta é comum a presença de pontos raros com dispersão variando de uniforme a difusa, bem como, a presença notória de eventos nulos em classes descontínuas considerando-se a amplitude total dos dados.

\section{CONCLUSÕES}

A pesquisa permitiu evidenciar as seguintes conclusões:

- O "UPLOT-DAP" pode ser utilizado em qualquer tipo de floresta, principalmente florestas naturais. Além disso, é bastante flexível e permite que o pesquisador escolha a escala mais conveniente para expressar a dispersão dos diâmetros.

- Mediante a utilização do "UPLOT-DAP", demonstrou-se que a tentativa de se fixar uma amplitude constante ou com variação constante crescente para os dados, poderia resultar em conclusões duvidosas sobre a estrutura diamétrica da floresta. Isto significa que a fixação de uma amplitude de $10 \mathrm{~cm}$ em todas as classes poderia trazer informações truncadas, mostrando um perfil da floresta diferente da realidade.

- Apesar de incorporar certo nível de subjetividade, a importância deste tipo de construção gráfica deverá se tornar evidente na medida em que a sua utilização for sendo implementada por pesquisadores que necessitem definir o número e a amplitude de classes diamétricas em uma população que não siga a distribuição normal e sobre a qual baseia-se a maioria dos métodos estatísticos.

- A pesquisa mostrou que se deve associar número de observações com a variabilidade total dos dados, a fim de que a probabilidade de determinados diâmetros ocorrerem no conjunto do seu espaço amostral, possa produzir resultados mais consistentes com os fatores físicos e biológicos que caracterizam a dinâmica de florestas naturais.

\section{AGRADECIMENTOS}

O autor agradece a colaboração do Prof. Dr. Jayme Cardoso, especialista em Neográfica, por suas sugestões valiosas e comentários, o que muito contribuiu para melhorar e enriquecer a qualidade final do presente artigo.

\section{REFERÊNCIAS}

BARROS, P. L. C. Estudo das distribuições diamétricas da floresta do planalto do Tapajós-Pará. Curitiba, 1980. Dissertação (Mestrado em Manejo Florestal) - Setor de Ciências Agrárias, Universidade Federal do Paraná. 123p.

BERTIN, J. A neográfica e o tratamento gráfico da informação. Curitiba, UFPR, 1986. 273p.

BONIN, S. Initiation à la graphique. Paris: Épi, 1983. 172p.

COSTA NETO, P. L. O. Estatística. São Paulo, Edgard Blücher Ltda, $17^{\text {a }}$ reimpressão, 1999, 264p.

CUNHA, U. S. Análise da Estrutura 
diamétrica de uma floresta tropical úmida da Amazônia Brasileira. Curitiba, 1995. Dissertação (Mestrado em Manejo Florestal) Setor de Ciências Agrárias, Universidade Federal do Paraná. 134p.

FUNDAÇÃO DE PESQUISAS FLORESTAIS DO PARANÁ-FUPEF. Inventário comercial da quadra de exploração $n^{0} 4$ da Floresta Nacional do Tapajós. Curitiba, 1984. 429p.

HOSHMAND, A. R. Statistical methods for enviroment \& agricultural sciences. CRC Press, New York, 2. ed. 1998. 439p.

HUSCH, B. ; MILLER, C. I. ; BEERS, T. W. Forest Mensuration. 3. ed. New York : Wiley, 1982. 402p.

IBAMA/PNUD. Plano de manejo florestal da Floresta Nacional do Tapajós, Pará, Brasil. Belém, 1997. 109p.
LÖETSCH, F. ; ZÖHRER, F. ; HALLER. K. E. Forest Inventory. BLV, München, 1973. v. 2. $469 \mathrm{p}$.

MOORE, D. A estatística básica e sua prática. Rio de Janeiro, Livros Técnicos e Científicos Editora S.A., 2000, 482p.

PRODAN, M. Forest Biometrics. Pergamon Press, New York. 1968. 447p.

STURGES, H. A. The Choice of Class Interval. J. Am. Stat. Assoc., v. 21, n. 65, 1926.

TUFTE, E. R. The Visual Display of Quantitative Information. United States of America, Graphcs Press, 2. ed. 2001, 197p. 